\title{
Kahlerian manifolds related in H-projective recurrent curvature killing vector fields with vectorial fields
}

\author{
U.S. Negi ${ }^{1}$, Preeti Chauhan ${ }^{2}$ \\ ${ }^{1,2}$ Department of Mathematics, \\ H.N.B. Garhwal University(A Central University), \\ S.R.T. Campus Badshahithaul,Tehri Garhwal - 249199, Uttarakhand, India. \\ 1usnegi7@gmail.com; 2preetichauhan1011@gmail.com
}

\begin{abstract}
Izumi and Kazanari [2], has calculated and defined on infinitesimal holomorphically projective transformations in compact Kaehlerian manifolds. Also, Malave Guzman [3], has been studied transformations holomorphic ammeters projective equivalentes. After that, Negi [5], have studied and considered some problems concerning Pseudo-analytic vectors on Pseudo-Kaehlerian Manifolds. Again, Negi, et. al. [6], has defined and obtained an analytic HP-transformation in almost Kaehlerian spaces. In this paper we have measured and calculated a Kahlerian manifolds related in H-projective recurrent curvature killing vector fields with vectorial fields and their holomorphic propertiesEinsteinian and the constant curvature manifoldsare established.Kaehlerian holomorphically projective recurrent curvature manifolds with almost complex structures by using the geometrical properties of the harmonic and scalar curvatures calculated overkilling vectorial fieldsare obtained.
\end{abstract}

Key words:H-projective, recurrent, curvature, Killing vector fields and Kaehlerian manifolds.

MSC 2010:53C15, 53B35,53A20.

\section{INTRODUCTION}

Kaehlerian manifolds in addition to complex hyper surfaces and other manifolds were measured implanted into special transformations with additive recurrent curvature properties and holomorphic projective correspondences and others. Considering $(M, g, F)$ as a Kaehlerian manifold of $2 n \geq 4$ dimension with $g=\left(g_{i j}\right)$ Riemannian metric and an almost-complex structure $F=F_{i j}$, where $F_{i j}=-F_{j i}$ and with Riemannian curvature tensor as well as Ricci tensor are:

$R_{k j i}^{h}=\partial_{k} \Gamma_{j i}^{h}-\partial_{j} \Gamma_{k i}^{h}+\Gamma_{k a}^{h} \Gamma_{j i}^{a}-\Gamma_{j a}^{h} \Gamma_{k i}^{a}$,

$P_{j i}=R_{a j i}^{a}(1.1)$

Now, the Ricci tensor and the $r=g^{b a} P_{b a}$ scalar curvature satisfy the following properties:

$\left[P_{j i}=F_{i}^{b} F_{i}^{a} P_{b a} \quad, H_{j i}=F_{j}^{a} P_{a i}\right.$,

$H_{j i}+H_{i j}=0$

$H_{j i}=F_{i}^{b} F_{i}^{a} H_{b a}, \quad F_{i}^{a} F_{i}^{h}=-\delta_{i}^{h}$,

$F_{j}^{a}=-i \delta_{j}^{a}$,

$g_{i j}=F_{i}^{a} F_{j}^{b} g_{a b}, \quad \nabla_{j} \tilde{F}_{i}=-\nabla_{i} \tilde{F}_{j}$,

$\left.\tilde{F}_{i}=F_{i} a F_{a}\right]$

Where, $F^{j i}=g^{j a} F_{a}^{i}, H_{j i}=F_{a}^{i} P_{a i}$.

The Lie operator derivatives in the vectorial field direction $\boldsymbol{x}$ for $R_{k j i}^{h}$ and $h_{j i}$ is represented respectively by, 


$$
\begin{aligned}
& L_{x} R_{k j i}^{h}=\nabla_{k} L_{x} \Gamma_{j i}^{h}-\nabla_{j} L_{x} \Gamma_{k i}^{h} y L_{x} \Gamma_{j i}^{h}= \\
& \nabla_{j} \nabla_{i} X^{h}+R_{a j i}^{h} X^{a}
\end{aligned}
$$

And if $X$ is a holomorphically projective transformation when:

$L_{X} \Gamma_{j i}^{h}=\delta_{i}^{h} V_{i}+\delta_{j}^{h} V_{i}-F_{j}^{h} F_{i}^{a} V_{a}-$ $F_{i}^{h} F_{j}^{a} V_{a},(1.5)$

Where $V=\left(V^{i}\right)$ is aexacting vector related to $X$.

Two metric $g=\left(g_{i j}\right)$ and $\bar{g}=$ $\left(\bar{g}_{i j}\right)$ defined on Kaehlerian manifolds $K_{n}$, they are holomorphic projective curvature correspondences if:

$\bar{\Gamma}_{k i}^{k}=\Gamma_{j i}^{k}+V_{i} \delta_{i}^{k}+V_{j} \delta_{i}^{k}-F_{j}^{k} \tilde{V}_{i}-F_{i}^{k} \tilde{V}_{j}$,

Where $\widetilde{V}_{I}=F_{i}^{a} V_{a}$.

Now, Tensors for harmonic and scalar curvature are defined on the Kahlerian manifold $K_{n}$ through the following relations:

$\nabla_{a} R_{k j i}^{a}=\nabla_{k} P_{j i}-\nabla_{j} P_{k i} R=g^{b a} P_{b a}$,

Respectively where $P_{j i}=R_{a j i}^{a}$ isthe Ricci tensor. The Laplacian of $\boldsymbol{f}$ is defined by:

$\Delta f=\nabla^{a} \nabla_{a} f=\Delta f$,

Where $f=\frac{1}{n+2} \nabla_{a} X^{a}$ with $f \in$ $C^{\infty}\left(K_{n}\right)$ and $V_{j}=\nabla_{j} f$.

The characteristic commutative relationship of $L_{X}$ and $\nabla$ for a curvature tensor $Y$ of $(1,2)$ type is given by:

$L_{X} \nabla_{k} Y_{j i}^{h}-\nabla_{k} L_{X} Y_{j i}^{h}=\left(L_{X} \Gamma_{k a}^{h}\right) Y_{j i}^{h}-$ $\left(L_{X} \Gamma_{k j}^{h}\right) Y_{a i}^{h}-\left(L_{X} \Gamma_{k j}^{a}\right) Y_{a i}^{h}-\left(L_{X} \Gamma_{k i}^{a}\right) Y_{j i}^{h}(1.9)$

Organism $X$ a holomorphically projective curvature transformation
If $X$ is a vectorial field then $X$ is a Killing vector field, If satisfies:

$L_{X} g_{j i}=0, \quad i, j=\overline{l, n} .(1.4)$

$\mathrm{a} V$ relatedvector then the following identities are satisfied Izumi, H. [1]:

$2 P_{i}^{a} V_{a}=-\nabla_{i}(\nabla f) \quad(1.10)$

$\nabla_{j} V_{i}=F_{i}^{a} F_{j}^{b} \nabla_{b} V_{a}$

$\nabla_{k} \nabla_{j} V_{i}=-F_{k}^{b} F_{j}^{a} R_{i a b}^{c} V_{c .}$

From Malave Guzman, [3] gives.

$P_{i j}=\overline{\bar{P}}_{i j}+\tau\left(V_{i j}-\bar{V}_{j i}\right)$,

Where $V_{i j}=\nabla_{i} \nabla_{j} f, \tau$ - parameter.

An $A_{n}=\left(K_{n}, \nabla\right)$ space is a Peterson Codazzi one if $\nabla_{k} P_{j i}=\nabla_{j} P_{k i}$. If $\nabla_{l} R_{i j k}^{h}=$ $V_{l} R_{i j k}^{h}$ it is a recurrent space where $V_{l} \neq$ 0 or it is an Einstein space if $P=\lambda g$ taking $S$ as the Ricci tensor and $g$ as the metric tensors and $\lambda$ as a parameter.

Theorem (1.1):If compact Kaehlerian manifold $K_{n}$ of dimension $n$ with a scalar curvature $R$ and it admits a holomorphically projective curvature transformation then the following equation is satisfied:

$\nabla f=-\frac{2 R}{n} f$ and $\quad P_{i}^{a} V_{a}=\frac{R}{n} V_{i}$

Proof. Since $A_{n}$ is a recurrent curvature space and compact Kaehlerian manifold $K_{n}$ admits an holomorphically projective curvature transformation then we get:

$g^{h i} \nabla_{b} \nabla_{j} X_{i}+g^{h i} R_{a b j i} X^{a}-V_{b} \delta_{j}^{h}-$

$V_{j} \delta_{b}^{h}+V_{a} F_{b}^{a} F_{j}^{h}+V_{a} F_{j}^{a} F_{b}^{h}=0$

Multiplying (1.14) by $g_{h k}$ and applying $\nabla^{b}$ it results that

$\nabla^{b}\left(\nabla_{b} \nabla_{j} X_{i}+R_{a b j i} X^{a}-V_{b} g_{j i}-V_{j} g_{b i}+\right.$ $\left.V_{a} F_{b}^{a} F_{j i}+V_{a} F_{j}^{a} F_{b i}\right)=0$

Now using Ricci's and Bianchi's identities we obtain

$\left(R_{a b j i}-2 R_{b j i a}\right) \nabla^{b} X^{a}-R_{a i} \nabla_{j} X^{a}+$

$R_{j}^{a} \nabla_{a} X_{i}-\left(\nabla_{a} R_{j i}\right) X^{a}=0$, 
Finally, by applying $\nabla^{j}$ the result is

$-2 \nabla_{i} R_{b a} \nabla^{b} X^{a}=0 \Rightarrow \nabla_{i} R_{b a} L_{X} g^{b a}=0$

$\Rightarrow-2 R F_{i}=n \nabla_{i}(\Delta f)$

$\Rightarrow \nabla_{i}(\Delta f)=-\frac{2 R}{n} f=\nabla_{i}\left(-\frac{2 R}{n} f\right)$

$\Rightarrow \Delta f=-\frac{2 R}{n} f$,

Due to $(n \Delta f+2 R f)$ be constant for being:

$\int_{M} \Delta f d \sigma=\int_{M} f d \sigma=0(1.19)$

A compact $K_{n}$ and $\mathrm{d} \sigma$ is a volumetric element of Kaehlerian manifold $K_{n}$.

Finally, we conclude that:

$\Delta f=-\frac{2 R}{n} f$.

Again, the expression is attained by above theorem (1.1), then we have the following:

Theorem(1.2): Let $X$ isa holomorphically projective curvature transformation with $\mathrm{aV}$ related vector then:

$L_{X} P_{j i}=-(N+2) \nabla_{j} V_{i}$

Proof. We have by the definition of $L_{X} R_{k j i}^{h}$ we have

$L_{X} P_{j i}=L_{X} R_{h j i}^{h}=\nabla_{h} L_{X} \Gamma_{j i}^{h}-\nabla_{j} L_{X} \Gamma_{h i}^{h}$

Because $\mathrm{X}$ is a holomorphically projective curvature transformation then:

$$
\begin{aligned}
\mathrm{L}_{X} P_{j i}=\nabla_{j} V_{i} & +\nabla_{i} V_{j}-F_{j}^{h} F_{i}^{a} V_{a}-F_{i}^{h} F_{j}^{a} V_{a} \\
& -n \nabla_{j} V_{i}-\nabla_{j} V_{i} \\
& +i n F_{i}^{a} \nabla_{j} V_{a}-\nabla_{j} V_{i}
\end{aligned}
$$

By considering the real part we obtain the desired result:

$$
\begin{aligned}
\mathrm{L}_{X} P_{j i}=-\mathrm{n} \nabla_{j} V_{i}-2 \nabla_{j} V_{i} \\
=-(n+2) \nabla_{j} V_{i}
\end{aligned}
$$

\section{KAHLERIAN MANIFOLDS RELATED IN H-PROJECTIVE RECURRENT CURVATURE KILLING VECTOR FIELDS WITH VECTORIAL FIELDS:}

Kaehlerian manifold to develop into a Peterson-Codazzi manifold under the assumption that the earlier is holomorphically projective then following theorem allocates:

Theorem (2.1): Let $K_{n}$ be a Kaehlerian manifold and $X$ be a holomorphically projective recurrent curvature killing vector field with arelatedto vectorial field $\mathrm{V}$ then:

$L_{X}\left(\nabla_{j} P_{k i}-\nabla_{k} P_{j i}\right)$

$=\left\{(n+2) R_{j k i}^{a}-P_{k i} \delta_{j}^{a}+P_{j i} \delta_{k}^{a}-\right.$

$\left.F_{i}^{a} H_{k i}+F_{k}^{a} H_{j i}+2 F_{i}^{a} H_{j k}\right\} V_{a .}(2.1)$

Proof.We have by the standard relation of commutation for a $(0,2)$ type tensor we obtain that:

$\left(L_{X} \nabla_{j} P_{k i}-L_{X} \nabla_{k} P_{j i}\right)-\left(\nabla_{j} L_{X} P_{k i}-\right.$

$\left.\nabla_{k} L_{X} P_{j i}\right)=\left(L_{X} \Gamma_{k i}^{a}\right) P_{j a}-\left(L_{X} \Gamma_{j i}^{a}\right) P_{k a}(2.2)$

But via hypothesis we consider $X$ as a holomorphically projective curvature transformation by using (1.5) then we gets:

$L_{X} \Gamma_{j i}^{a}=\delta_{j}^{a} V_{i}+\delta_{i}^{a} V_{j}-F_{j}^{a} F_{i}^{h} V_{h}-$ $F_{i}^{a} F_{j}^{h} V_{h}(2.3)$

Additionally, treating to theorem (1.2),

$L_{X} P_{j i}=-(n+2) \nabla_{j} V_{i}$

And a rationally, we obtain $L_{X} \Gamma_{k i}^{a}$ and $L_{X} P_{k i}$.

Through Substituting (2.3) and (2.4) in (2.2), we get:

$\left(L_{X} \nabla_{j} P_{k i}-L_{X} \nabla_{k} P_{j i}\right)-\left(\nabla_{j}[-(n+\right.$

2) $\left.\left.\nabla_{k} V_{i}\right]-\nabla_{k}\left[-(n+2) \nabla_{j} V_{i}\right]\right)$

$=\left(\delta_{k}^{a} V_{i}+\delta_{i}^{a} V_{k}-F_{k}^{a} F_{i}^{h} V_{h}\right.$

$\left.-F_{i}^{a} F_{k}^{h} V_{h}\right) P_{j a}$

$-\left(\delta_{j}^{a} V_{i}+\delta_{i}^{a} V_{j}-F_{j}^{a} F_{i}^{h} V_{h}\right.$

- $\left.F_{i}^{a} F_{j}^{h} V_{h}\right) P_{k a}$

Through some operation and using generalization, we bring to a close that: 


$$
\begin{aligned}
\left\{(n+2) R_{j k i}^{a}-\right. & P_{k i} \delta_{j}^{a}+P_{j i} \delta_{k}^{a}-F_{i}^{a} H_{k i} \\
& \left.+F_{k}^{a} H_{j i}+2 F_{i}^{a} H_{j k}\right\} V_{a} \\
& =L_{X}\left(\nabla_{j} P_{k i}-\nabla_{k i} P_{j i}\right)
\end{aligned}
$$

As of there on various submissions of the earlier outcomes will be given that:

A.If $\nabla_{j} P_{k i}=\nabla_{k} P_{j i}$ then $K_{n}$ is KaehlerPeterson-Codazzi manifolds and $\left\{(n+2) R_{j k i}^{a}-P_{k i} \delta_{j}^{a}+P_{j i} \delta_{k}^{a}-F_{i}^{a} H_{k i}+\right.$ $\left.F_{k}^{a} H_{j i}+2 F_{i}^{a} H_{j k}\right\} V_{a}=0 .(2.5)$

Therefore, first significance is a KaehlerPeterson-Codazzi manifold has a harmonic curvature in view of the fact that:

$\nabla_{j} P_{k i}=\nabla_{k} P_{j i} \Leftrightarrow \nabla_{a} R_{j k i}^{a}=0$.

Also, second significance is a Kaehler-Peterson-Codazzi manifold is an Einsteinian manifold if the previous has a constant scalar curvature. Exactly through multiplying $g^{k i}$ into (2.5) then we get:

$$
\begin{aligned}
&\left\{(n+2) g^{k i} R_{j k i}^{a}-R \delta_{j}^{a}+g^{a i} P_{j i}\right. \\
&-F_{i}^{a} g^{k i} H_{k i}+F g^{k i} H_{j i} \\
&\left.+2 F_{i}^{a} g^{k i} H_{j k}\right\} V_{a}=0 .
\end{aligned}
$$

While $V_{a} \neq 0$ and budding the three previous terms we comprise,

$$
\begin{aligned}
(n+2) g^{k i} R_{j k i}^{a} & -R \delta_{j}^{a}+g^{a i} P_{j i} \\
& -F_{i}^{a} F_{k}^{b} g^{k i} P_{b i} \\
& +3 F_{k}^{a} F_{j}^{b} g^{k i} P_{b i}=0,
\end{aligned}
$$

Next to creation the reduction $a=F$ and estimate starting 1 to $n$ then we get hold of:

$g_{k i}(n R+2 R-n R+R) 3 P_{k i}$,

Hence like this, we complete that $P_{k i}=$ $\frac{R}{n} g_{k i}$. then further expressions the Kaehler- Peterson-Codazzi manifold is an Einsteinian manifold.

B.If Kaehlerian manifold $K_{n}$ is a recurrent curvature manifold, then:

$$
\begin{aligned}
& (n+2) R_{j k i}^{a} V_{a}-L_{X}\left(R_{j k i}^{a} V_{a}\right)=P_{k i} \delta_{j}^{a}- \\
& P_{j i} \delta_{k}^{a}+F_{j}^{a} H_{k i}-F H_{j i}-2 F_{i}^{a} H_{j k} V_{a .}(2.6)
\end{aligned}
$$

Hence, significance will be, if Kaehlerian manifold $K_{n}$ is a harmonic curvature and $W=\left\{V=\left(V^{i}\right): V \neq 0\right\}$ with $V_{j} V^{k}=$ ||$V||^{2}$ if $(j=k)$ and $V_{j} V^{k}=0$ if $(k \neq$ $j$ ), Then Kaehlerian manifold $K_{n}$ has a null scalar curvature.

In point of fact, if Kaehlerian manifold $K_{n}$ confesses a harmonic curvature then building the reduction $l=a$ and summing up from 1 to $n$ in the relation:

$\nabla_{l} R_{j k l}^{a}=R_{j k l}^{a} V_{l}$

We get,

$\nabla_{l} R_{j k i}^{a}=R_{j k i}^{a} V_{l} \Rightarrow R_{j k i}^{a}=0$.

From (2.1) we get,

$$
\begin{aligned}
P_{k i} V_{j}-P_{j i} V_{k}+ & H_{k i} \tilde{V}_{j}-H_{j i} \tilde{V}_{k}-2 H_{j k} \tilde{V}_{i} \\
& =0
\end{aligned}
$$

And multiplying the earlier relation by $g^{k i}$ it affects that:

$$
\begin{aligned}
g^{k i} P_{j i} V_{k}-g^{k i} P_{k i} V_{j}+g^{k i} H_{j i} \tilde{V}_{k} \\
-g^{k i} H_{k i} \tilde{V}_{j}-2 g^{k i} H_{k j} \tilde{V}_{i} \\
=0
\end{aligned}
$$

Hence

$P_{j}^{k} V_{k}-r V_{j}=H_{j}^{k} \tilde{V}_{k}$,

Somewhere in through relating $V^{j}$ itproducts that $r=0$. Thus we finish off that the Kaehlerian manifold $K_{n}$ is natural.

Theorem (2.2):If exist Einstein dense Kaehlerian manifold $K_{n}=(M, \nabla)$ and $\bar{K}_{n}=$ $(M, \bar{\nabla})$, with metric $g=\left(g_{i j}\right)$ and $\bar{g}=$ $\left(\bar{g}_{i j}\right)$ holomorphic projective curvature identical, therefore we contract an term that recounts the scalar curvature $\mathrm{R}$ and $\bar{R}$.

Proof. We have using Martinez and Ramirez [4]. 
$P_{i j}=\bar{P}_{i j}+\tau\left(V_{i j}-\bar{V}_{j i}\right)$,

Then in addition to like Einstein

manifolds:

$$
\begin{aligned}
& P_{i j}=c_{1} g_{i j}, \quad \bar{P}_{i j}=c_{2} \bar{g}_{i j} \text { or } \\
& P_{i j}=\frac{R}{n} g_{i j}, \quad \bar{P}_{i j}=\frac{\bar{R}}{n} \bar{g}_{i j,}
\end{aligned}
$$

It should be

$\frac{R}{n} g_{i j}=\frac{\bar{R}}{n} \bar{g}_{i j}+\tau\left(V_{i j}-\bar{V}_{j i}\right), \quad \tau \in C$,

After that, concerning $g^{i j}$ gets the outcome:

$R=\frac{\bar{R}}{n} g^{i j} \bar{g}_{i j}+\tau\left(g^{i j} V_{i j}-g^{i j} \bar{V}_{j i}\right)$

orR $=$

$\frac{\bar{R}}{n} g^{i j} \bar{g}_{i j}+\tau\left(\|V\|-g^{i j} \bar{V}_{j i}\right)$ or $R g_{i j}=$

$\frac{\bar{R}}{n} \bar{g}_{i j}+\tau\left(\|V\| g_{i j}-\bar{V}_{i j}\right)$

Again concerning now $\bar{g}^{i j}$ gets the results:

$R g_{i j}=\bar{R} \bar{g}_{i j}+\tau\|V\| g_{i j}\left(-\|\bar{V}\| \bar{g}_{i j}\right)$,

As ofat this time: $(R-\tau\|V\|) g_{i j}=$ $(\bar{R}-\tau\|\bar{V}\|) \bar{g}_{i j}$,in that case

$\frac{(R-\tau\|V\|) g_{i j}}{(\bar{R}-\tau\|\bar{V}\|) \bar{g}_{i j}}=\frac{\operatorname{det}\left(\bar{g}_{i j}\right)}{\operatorname{det}\left(\bar{g}_{i j}\right)}$

Using by Malave Guzman [3] has studied Transformationes holomorficamemte proyectivas equivalentes article evidence then we have:

In $\sqrt{\frac{\operatorname{det}\left(\bar{g}_{i j}\right)}{\operatorname{det}\left(\bar{g}_{i j}\right)}}=(n+2) h, \quad h \in C^{\infty}\left(K_{n}\right)$

Thuswe conclude and find the relation:

$$
\begin{aligned}
(R-\tau\|V\|)= & (\bar{R} \\
& -\tau\|\bar{V}\|) \exp [2 h(n+2)] .
\end{aligned}
$$

Theorem (2.3):We obtain an expression that calculate the Ricci tensor in a dense Kahlerian manifold $K_{n}$ confessing holomorphic projective curvature transformations with related vector $\mathrm{V}$, if $A_{n}$ this is recurrent curvature.
Proof: We have in this case:

$$
\nabla_{k} R_{l j i}^{h}=R_{l j i}^{h} V_{k}
$$

By making the contraction $a=j$ and adding up from 1 to $n$ we get:

$\nabla_{k} P_{j i}=P_{j i} V_{k},(2.8)$

Although, we have

$\nabla_{k} P_{j i}=\partial_{k}\left(P_{j i}\right)-\Gamma_{k j}^{a} P_{a i}-\Gamma_{k i}^{a} P_{j a}$.

Concerning $g^{i j}$ then outcome is:

$\nabla_{k} P_{j i}=\partial_{k}\left(P_{j i}\right)(2.9)$

After then using (2.8) and (2.9) we find the results:

$\partial_{k}\left(P_{j i}\right)-P_{j i} \partial_{k} f=0$.

Therefore, the solution of this partial differential equation is the Ricci tensor, hence is achieved the scalar curvature tensor.

\section{REFERENCES}

1.H. Izumi, A remark on infinitesimal holomorphically projective transformations, Mat. Japonica, 39, pp. 47-60, 1994.

2.H. Izumi, and Y. Kazanari. On infinitesimal holomorphically projective transformations in compact Kaehlerian manifolds, Hokkaido Math. J., 8, pp. 6579, 1970.

3.R. Malave Guzman. Transformationes holomorficamemteproyectivas equivalentes, Department Mathematic de la Universidad de oriente, (tesis de maestria) 2007.

4.R. Martinez,andR. Ramirez. Lyra spaces, their application to mechanics, Jadronic, J, 12, pp. 123-236, 1992.

5.U.S. Negi.Some problems concerning Pseudo-analytic vectors on PseudoKaehlerian Manifolds. AARJMD, vol. 1, issue 17, pp. 106-113, 2014. 
U.S. Negi et al., International Journal of Emerging Trends in Engineering Research, 9(4), April 2021, 435 - 440

6. U. S. Negi et., al. An analytic HPtransformation in almost Kaehlerian spaces., Aryabhatta Journal of Mathematics \& informatics, Vol. 11, No. 1, pp. 103-108, 2019. 